\title{
Reform and practice for photoelectric specialty experimental teaching based on virtual simulation experiment platform
}

Yan Ye, Qingsong Lv, Maocheng Wu, Yishen Xu, Jihua Gu

Yan Ye, Qingsong Lv, Maocheng Wu, Yishen Xu, Jihua Gu, "Reform and practice for photoelectric specialty experimental teaching based on virtual simulation experiment platform," Proc. SPIE 10452, 14th Conference on Education and Training in Optics and Photonics: ETOP 2017, 1045245 (16 August 2017); doi: 10.1117/12.2266489

SPIE Event: 14th Conference on Education and Training in Optics and Photonics, ETOP 2017, 2017, Hangzhou, China 


\title{
Reform and practice for photoelectric specialty experimental teaching based on virtual simulation experiment platform
}

\author{
Yan Ye, Qingsong Lv, Maocheng $\mathrm{Wu}$, Yishen $\mathrm{Xu}^{*}$, Jihua $\mathrm{Gu}$ \\ College of Physics, Optoelectronics and Energy, Soochow University, Suzhou 215006, China
}

\begin{abstract}
In view of some problems about the traditional photoelectric specialty experimental teaching process, such as separation of theoretical teaching and practical teaching, immobilization of experimental teaching contents, low quality of experiments and no obvious effect, we explored and practiced a new experimental teaching model of "theoretical teaching, virtual simulation and physical experiment", which combined the characteristics of photoelectric information science and engineering major and the essential requirements of engineering innovation talents cultivation. The virtual simulation experiment platform has many advantages, such as high performance-to-price ratio, easy operation and open experimental process, which makes virtual simulation combine physical experiment, complete each other with virtual for practical. After the users log into the virtual simulation experimental platform, they will first study the contents of the experiment, clarify the purpose and requirements of the experiment, master the method of using the instrument and the relevant notes, and then use the experimental instruments provided by the platform to build the corresponding experimental system. Once the experimenter's optical path is set incorrectly or the instrument parameters are set incorrectly, the error or warning message will be automatically triggered, and the reference information will be given instructing the student to complete the correct experimental operation. The results of our practice in recent years show that the teaching reform of the photoelectric specialty experiments has not only brought great convenience to the experimental teaching management, broadened the students' thinking and vision, enhanced the students' experimental skills and comprehensive qualities, but also made the students participate in the experiment with their enthusiasm. During the construction of experiment programs, the students' engineering practical ability and independent innovation awareness has been improved greatly. In the next time, based on the development trend of optoelectronic discipline and our own major characteristics, we will further perfect and enrich the construction of virtual simulation experimental platform and continuously improve the quality of experimental teaching.
\end{abstract}

Keywords: virtual simulation, photoelectric specialty experiment, talents cultivation, experimental teaching

\section{INTRODUCTION}

Experimental teaching is an effective way to consolidate and deepen the understanding of theoretical knowledge, and it plays an important role in cultivating high-quality talents with innovative consciousness. However, there are a series of problems about the traditional photoelectric specialty experimental teaching process, such as separation of theoretical teaching and practical teaching, immobilization of experimental teaching contents, low quality of experiments and no obvious effect, which can't activate students' interest in learning and excavate the potential of students, and easily lead to the lack of practical ability of students. Therefore, the promotion of experimental teaching reform is a vital part in the current education and teaching reform.

In recent years, the rapid development of virtual laboratories, integrate the simulation of graphics and images, virtual reality, network, real-time computer control and other technologies, which could create a virtual experimental environment including laboratory equipment, experimental objects and experimental information resources. The virtual laboratories have the advantages of openness, interaction, safety and high cost-effective, and could effectively avoid the limitation such as shortage of resources, limited equipment and fixed time in traditional experimental teaching mode ${ }^{[1-3]}$. At the same time, students in the virtual simulation experimental teaching platform can freely learn theoretical knowledge, self-concept, design the experimental program to confirm the knowledge learned in the classroom, which are the lacks of traditional experimental teaching model.

*xys2001@suda.edu.cn 
In this paper, taking the course of "comprehensive experiment of optical information" as an example, the main problems of traditional optical experiment teaching are analyzed. With the help of virtual simulation platform and based on the "open teaching" educational philosophy, a new experimental teaching model of "theoretical teaching, virtual simulation and physical experiment" was explored and practiced, which combined the characteristics of photoelectric information science and engineering major and the essential requirements of engineering innovation talents cultivation.

\section{SHORTCOMINGS OF TRADITIONAL PHOTOELECTRIC EXPERIMENTS}

Experimental teaching is an important platform for integrating theory with practice, improving students' ability of analyzing problems, solving problems and practicing ability. However, for the traditional optical experiment teaching, the experiment content is basically to consolidate and deepen the theoretical teaching, and only requires the students to deepen the understanding of theoretical knowledge through the experiments. Therefore, traditional optical experiment teaching not only can't effectively mobilize the enthusiasm of students, but also is detrimental to the cultivation of students' comprehensive quality. The main questions can be summarized as follows.

\subsection{Separation of theoretical teaching and practical teaching}

Theoretical knowledge is the basis of experimental research, so learning of the relevant professional theory is the primary condition of the experiment. However, the "comprehensive experiment of optical information" course covers the basic knowledge of classical physics optics such as optical interference, diffraction and polarization, and incorporates many new knowledge points of modern optics such as optical information processing, holography and optoelectronics ${ }^{[2]}$. Therefore, the separation of theoretical teaching and practice teaching and the non-synchronization phenomenon in the optical experiment teaching process are widespread ${ }^{[3]}$. Students start experimenting without understanding the experimental principles, inevitably leading to great reduction of experimental teaching effect.

In addition, the teacher who teaches the theory is usually not the same as the teacher who directs the experiment. The former is only responsible for the teaching of relevant theoretical knowledge, and not clear what specific instruments or equipment in the laboratory to verify the corresponding theory; the latter is only responsible for the specific operation of the experimental explanation, regardless of whether the students have mastered the theory of the experiment basis. This not only reduces the enthusiasm of students, but also wastes valuable experimental resources.

\subsection{Immobilization of experimental teaching contents}

For a long time, experimental teaching has been designed to validate one aspect of teaching content. For optical experiments, because theoretical knowledge involved is generally more classic, so the experimental contents usually are unchanged for several years or even ten years, resulting in the simplification and immobilization of the experimental teaching contents. Moreover, the traditional verification-based experiments often are firstly explained about the experimental principles, operational processes, data collection and processing steps by the teacher, then the teacher demonstrates the experimental operation, and finally students repeat them again to complete the experimental tasks. Optical experiment teaching usually is only auxiliary and supplementary to the theoretical teaching, and not as a curriculum independent of the theoretical course, which causes students do not pay enough attention to the experimental curriculum, lack of time and energy, and even regard the experimental curriculum as a learning burden.

\subsection{Low quality of experiments}

Optical experiment operation is more difficult, the adjustment of experimental equipment requires some skills and experience. Moreover, the limited time in the experimental classroom and the limitations of the experimental equipment, the experimental site requires students to complete the experiment in the specified classroom time. Therefore, the conventional approach is that the experimental teacher should first adjust the experimental equipment in place before the experiment teaching, and then students only need to observe the phenomena, follow the prescribed order and record experimental data in the specific experiment. The drawback of this operation is that on the one hand, the workload of the experimental teacher is increased, and it takes a lot of time to carry out the calibration of the experimental instrument and the adjustment of the optical path; On the other hand, in the whole process of experiment, the student has only got a few simple experimental data, which could result in poor quality of experiment and the unsatisfactory experimental effect, and couldn't achieve the desired experimental teaching objectives.

In addition, the experimental data of many conventional optical experiments often are acquisited by the eyes, which is easy leading to experimental data deviation or even wrong. 


\section{ADVANTAGES OF VIRTUAL SIMULATION EXPERIMENT TEACHING}

The virtual simulation experiment teaching is an experimental teaching method based on computer technology and network technology, so that the experimenters could complete the scheduled experimental project or opening experiment in the near real experimental environment. Virtual simulation labs have obvious advantages in terms of time, space, and lab management:

- The virtuality of experimental equipment. Although the experimental equipment operated in the virtual simulation experiment platform can be seen and felt, but they do not exist in fact, just a device that is simulated by a computer depending on the simulation of the actual object. This will not only reduce the cost of the construction, maintenance of the laboratory and the cost of the experiment, but also improve the utilization of the laboratory. In addition, virtual simulation experiments could provide an effective solution to the optical phenomena and experimental results, which cannot be obtained directly by some traditional optical experiments.

- The simplicity of experimental operation. In the virtual simulation experiment platform, based on the graphical user interface, some complex, precise experimental operation can be achieved only through the corresponding control of mouse and button. It is easy to change the corresponding experimental parameters and experimental conditions for the experimenter, thus simplifying the experimental operation, saving the experimental time and improving the efficiency and quality of the experiment. In addition, in the virtual experimental environment, part of the teacher's counseling can be done through the simulation software itself, so that the experimental teachers can be free from the complex experimental guidance work to a certain degree.

- The openness of the experimental process. As an open network experiment platform, virtual laboratory is not limited by the time and space of traditional experiment, the experimenter can do all kinds of experiments at any time and place ${ }^{[4]}$. At the same time, it can also achieve collaboration among many persons. Therefore, it is convenient for the students to design a variety of opening experiments and to verify their own views and assumptions, so as to arouse the students' autonomous learning consciousness. Moreover, students can learn the experimental operation, experience the discovery process of theoretical knowledge, thus enhance students' experimental skills and comprehensive qualities.

\section{SPECIFIC MEASURES FOR REFORM OF OPTICAL EXPERIMENT TEACHING}

\subsection{Reform of teaching mode}

Except for carrying out experimental operation teaching, the increasing teaching of relevant theoretical knowledge is essential for solving the problem of separation between theory teaching and experimental teaching, that is to say, replacing the partial experimental hours of operation with virtual simulation. For the benefits of virtual experiment platform is not restricted by time and space compared to the traditional experiments, virtual simulation combines the "concentrated experiments in the lab" and "extracurricular free experiments". The students are required to complete the corresponding experimental tasks before the deadline. Aiming at the phenomenon of "learned contents can't be used in practice", "learned contents can't be used accurately" and the lack of students' attention to the traditional experiment teaching, it is necessary to make full use of the virtual experimental platform advantages, reforming the original experimental teaching evaluation system and taking experimental design capability included in experimental assessment. The enthusiasm of students to participate in the experiment is fully mobilized and the students are guided to learn in experiment design. As a consequence, the comprehensive practical ability and innovation ability of student will be fully promoted.

In practice, students are required to complete the relevant theoretical knowledge and experimental learning process involved in the experiment on the virtual simulation experiment platform before each physical experiment. When doing experiments, students are required to complete the corresponding experimental operation based on the virtual simulation platform, and then submit the corresponding experimental report after the experimental data processing. In the course of assessment, the students are required within the specified time according to the experimental project to design their own experimental program, and then select the appropriate instrument from the instrument library to build experimental optical path and complete the experiment. The experimental teachers evaluate the students' experimental scheme and operation process through the experimental data interface. 


\subsection{Construction of virtual experiment platform}

Specific use of the virtual experimental platform is shown in Figure 1. After the experimenter logs into the virtual simulation experiment platform, it is necessary to study the experiment contents, clarify the purpose and requirements of the experiment, and master the use of the experimental equipment and related precautions, as shown in Figure 2. Then, by using a variety of experimental instruments provided by the instrument library, and accordance with the experimental steps, the corresponding experimental system is built as shown in Figure 3.

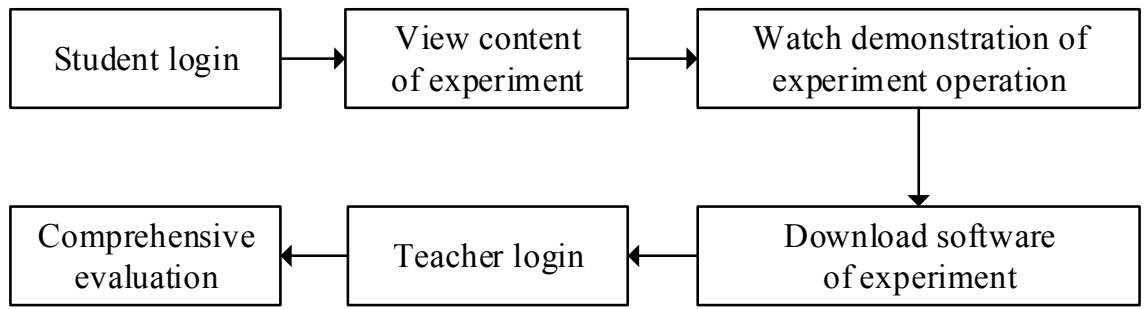

Figure 1. Specific use of the virtual experimental platform.

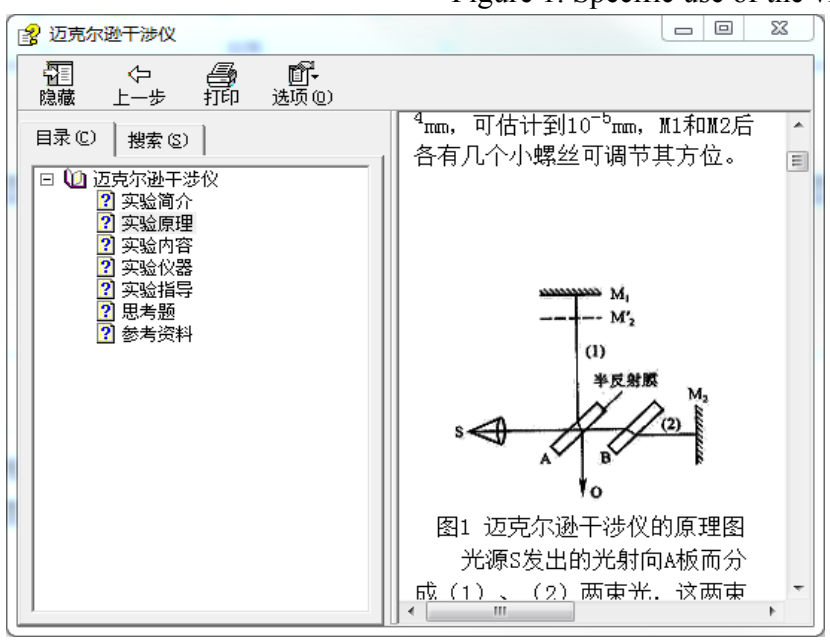

Figure 2. Experimental content learning window.

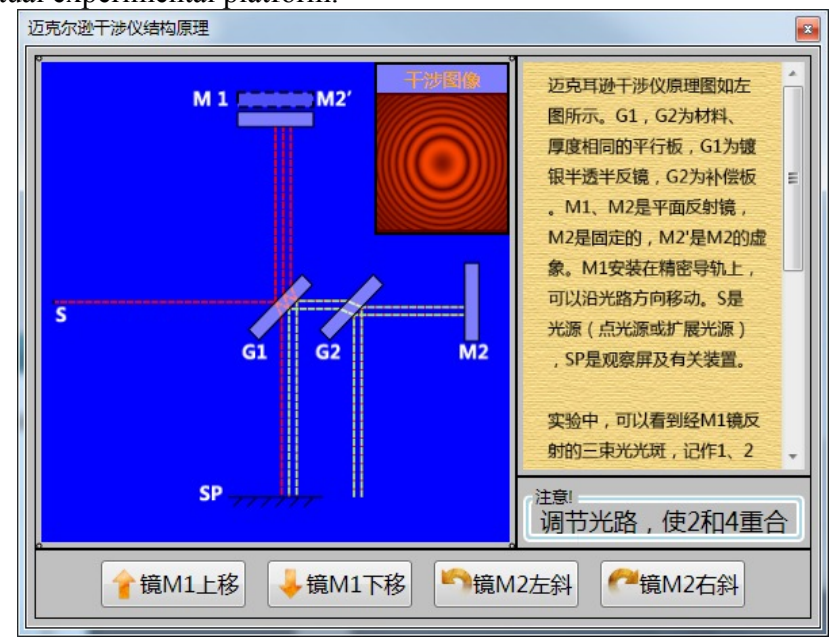

Figure 3. Experimental principle demonstration window.

Among them, the simulation experiment platform built-in Figure 5 shows a variety of optical components, and classification management, the experimenter through the search function can quickly find the required components. Moreover, when the error of the optical path is set up or the parameters of the instrument are not set correctly, the error message will be automatically triggered, and a reference message similar to Figure 6 will be given to guide the student to complete the correct experimental operation.

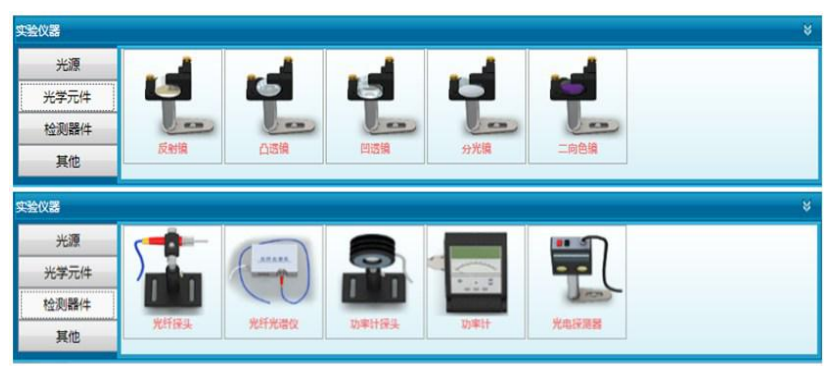

Figure 5. Optical component management window.

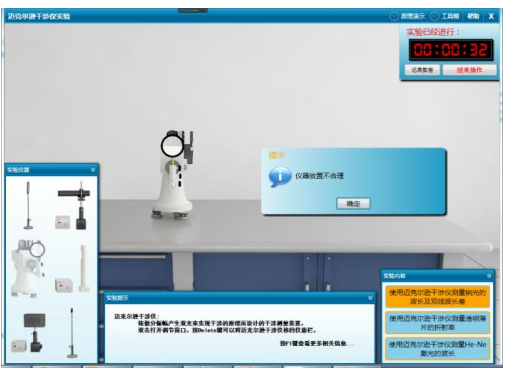

Figure 6. Suggestive window of experimental error.

In addition, the construction of the optical virtual simulation platform also has the following functions:

- Free definition of interface style. The window scale of the experimental scene could be adjusted freely, and the various functional modules can also be packed up or expanded according to actual needs. In addition, the windows of instrument are simple and beautiful, high degree of authenticity and easy to operate. 
- Real-time recording of experimental status. Throughout the experimental operation, not only the scene of the various instruments in all the state information can be viewed in real time, but also the preservation and recovery of experimental status could be provided in real time. At the same time, the platform has some built-in programmable calculators and functions fitting function, which could bring great convenience for the experimental data processing.

- Experimental report customization and export. As the experimental platform has customized a number of project reporting templates, so when the completion of the current experimental project, students only need to follow the prompt information, import the experimental data and analysis results into the experimental template, and the platform can automatically generate experimental report documents.

\section{CONCLUSIONS}

There are two advantages of the development of the proposed virtual simulation experiment teaching mode. On the one hand, the effect of experimental teaching is promoted by the combination of virtual experiment and reality experiment. On the other hand, advanced scientific research achievements can be integrated, the relationship between experimental teaching and scientific research will get close, high quality resources of subject serving teaching will be implemented. Meanwhile, the development of virtual simulation experiment teaching mode brings much convenience to the management of experiment teaching. The logic mind and horizons of students are broadened by the proposed teaching mode. The experimental ability and comprehensive quality of students are enhanced. The engineering practice ability and independent innovation consciousness of students will be well tempered in the process of designing the experiment plan. In the next time, based on the development trend of optoelectronic discipline and our own major characteristics, we will further perfect and enrich the construction of virtual simulation experimental platform and continuously improve the quality of experimental teaching.

\section{ACKNOWLEDGEMENTS}

This work was supported by 2015 teaching reform research project in higher education of Soochow University (youth project) and by $3^{\text {th }}$ teaching research project about hot and difficult issues in higher education for specialty in optoelectronic information science and engineering of the Chinese colleges and universities.

\section{REFERENCES}

[1] Lin, Y. F., Zheng, X. D., Zhang, D. X., Li, H. F., Liu, X. D. and Liu, X., "Construction plan of a virtual laboratory for the course of 'composite experiments of optical information science'," Optical Technique, Vol. 35, 182-184 (2009).

[2] Li, H. F., Zhang, D. X., Zheng, Z. R., Zheng, X. D. and Lin, Y. F., "Reform and consideration of the course 'comprehensive experiments of optical information science' " Optical Technique, Vol. 35, 175-177 (2009).

[3] Goldberg, H., "What is virtual instrumentation," IEEE Instrumentation \& Measurement Magazine, 10-13 (2000).

[4] Dong, J., Zhang, L., Sun, L. and Long, Z. J., "Innovative talents training oriented virtual experimental teaching reform - take 'design and manufacture of MEMS' as an example," Journal of Zhejiang University of Technology (Social Science), Vol. 15, No. 1, 100-104 (2016). 